EESTI NSV TEADUSTE AKADEEMIA TOIMETISED. VII KOIDE

BIOLOOGILINE SEERIA. 1958, NR. 1

ИЗВЕСТИЯ АКАДЕМИИ НАУК ЭСТОНСКОЙ ССР. ТОМ VII

СЕРИЯ БИОЛОГИЧЕСКАЯ. 1958, 수 1

\title{
MARKMEID EESTI NSV MADALSOODE TSIKAADIDE FAUNAST
}

\section{J. Vilbaste}

Kuigi eelmise sajandi keskel, tänu Tartu ülikooli tolleaegse professori G. Flori tegevusele, võis pidada Eestit tsikaadide suhtes üheks parimini uuritud alaks terves maailmas, on nimetatud putukaterühm meil viimasel ajal väga vähe uurijate tähelepanu köitnud. Pärast Flori monograafiat «Die Rhynchoten Livlands» (1861) on ilmunud selle kohta rida väiksemaid märkusi mitmetes vastavates regionaalsetes töödes ja vaid üks suurem töö rootsi entomoloogilt Ossiannilssonilt (1951), kes töötas läbi prof. H. Habermani poolt määramiseks saadetud materjali.

Selletōttu osutusid paljud Eesti madalsoodest kogutud tsikaadide liigid meile uuteks. Kuna tsikaadide süstemaatika on eriti viimastel aastakümnetel tugevasti arenenud, on mitmed vanemate autorite liigid osutunud liikide rühmadeks, millest on kirjeldatud suur hulk uusi liike. Eesti madalsoodest ja soode kuivendatud aladelt kogutud 99-st tsikaadiliigist osutusid 35 liiki ja üks vorm uuteks Eesti NSV-s ning neist 9 liiki ja üks vorm uuteks kogu Nõukogude Liidus. Alljärgnevalt esitatakse üksikud liigid süstemaatilises järjekorras koos märkustega liigi ökoloogia ja leviku kohta. ${ }^{1}$ Kōik toodud liigid, välja arvatud Aphrodes striatus (F.), on uuteks Eesti NSV-s. Uued liigid Nõukogude Liidus on tekstis märgitud tärnikesega liigi nimetuse ees.

1. Oliarus le porinus (L.), 1761. Leitud mitmest madalsoost (Patsu-Laiküla soo, Suursoo ja Epu soo). Näib Eesti NSV oludes toituvat pilliroost. Kirjanduse andmeil toituvat peale selle ka jäneslillest ja lugadest. Siberi.

Levinud peaaegu üle kogu Euroopa, tungides põhjas Lõuna-Soomeni, ja üle Lääne-

2. Kelis i a rib a uti (W. Wagn.), 1938. Eestis esineb umbes pooltes madalsoodes. Kohati on üsna arvukas. Nii esines Saaremaal Järise soos pruuni sepsika ja lubikaniidul sajalöögilises kahapüügis 248 isendit.

Varem on segi aetud liigiga $K$. guttula Germ. Ka Flori kogus on rida isendeid viimase nimetuse all. (Leiukohti ei ole kahjuks võimalik kindlaks teha, sest osa kataloogist on kadunud.)

K. ribauti levik on ebaselge. Kindlaks on ta tehtud Soomest, Rootsist, Poolast ja Tšehhoslovakkiast.

Nōukogude Liidu teistest osadest on autoril teada rida leide vaid Karjala ANSV-st (Lindberg, 1947). Veel mainib teda Zahvatkin oma avaldamata Moskva oblasti tsikaadide nimekirjas. Arvatavasti on liik Nõukogude Liidus üsna laia levikuga. ${ }^{2}$

3. Kelisia vittipennis (J. Shlb.), 1871. Esineb koos eelmise liigiga paljudes madalsoodes, kus elab (kirjanduse andmeil) villpeal või tarnadel.

Esineb Flori kogus samuti $K$. guttula nimetuse all.

Levik Nōukogude Liidus on ebaselge, sellepärast et teda on varem segi aetud teiste sama perekonna liikidega.

*4. Delph a codes pilos us (Hpt.), 1935. Eestist leitud Avaste ja Patsu-Laiküla soost ning Suursoost ja Emajōe suudmeala soodest. Arvestades liigi varjatud eluviisi on tōenäoline, et teda esineb veel mitmes teiseski soos.

Levik ebaselge. Seni leitud Rootsist, Pōhja-Saksamaalt, Poolast, Inglismaalt. Nōukogude Liidust pole peale Eesti NSV teada ühtki leidu; küll aga esines ZIN'i kogus Tadžikistanist üksik eksemplar, mis väliselt sarnanes täiesti selle liigiga.

Kuna selle liigi genitaalide jooniseid pole varem avaldatud, siis esitame need joonistel $1-4$.

${ }^{1}$ Nimestik leitud liikide kohta on ilmunud autori ühes varasemas, peamiselt soonokaliste ökoloogiat käsitlevas töös (1955). Käesolev kirjutis on mõeldud viimase täienduseks.

${ }^{2}$ Läbi vaadanud NSV Liidu TA Zooloogia Instituudi kogudes $K$. guttula isendid, ei leidunud nende hulgas ühtki ōiget guttula't, vaid kōik olid musta joonega esisäärtel, s. o. kuulusid kas ribauti, monoceros Rib. vōi mõne kirjeldamata liigi hulka. 

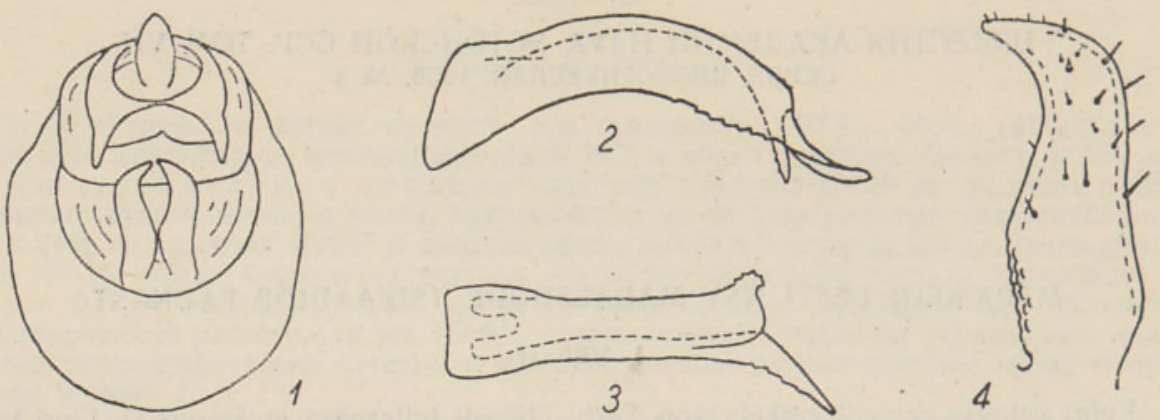

Joon. 1. Delphacodes pilosus (Hpt.). 1 - isase genitaalsegment tagantvaates (suurendus ca $10 \times), 2$ - aedeagus lateraalselt $(200 \times), 3-$ aedeagus dorsaalselt $(200 \times), 4$ - stylus tagantvaates $(200 \times)$.

5. Delphax pulchellus (Curt.), 1833. Oksik ô eksemplar leiti Kurtnast Nōmme küla lähedalt väikeselt soolt 27. VII 1954, Linnavuori (1952) järgi halobiont, kes Soomes elab vaid mererannikul. Elab pillirool.

A Laia levikuga - Euroopast kuni Turkestanini.

*6. Chlorion a glaucescens (Fieb.), 1866. 2 \& eksemplari leitud koos eelmise liigiga. Linnavuori (1952) järgi pillirool elav halofiil, keda on Soomes esinenud seni vaid mererannikul.

Levinud peaaegu kogu Lääne-Euroopas. Nõukogude Liidust pole autoril peale Eesti NSV teada ühtki leidu.

7. C hlo ri o n a sma r a g d u la (Stål), 1853. Oksikud leiud Lääne-Eesti madalsoodest - Avaste soost ja Suursoost. Linnavuori (1952) järgi tüüpiline sooliik, keda pole leitud mere äärest, kus samuti esineb tema toitetaim - pilliroog.

Kirjanduse andmeil levinud Lääne-Euroopast Turkestanini.

8. Stenocranus fuscovittatus (Stål), 1858. Leitud Avaste soost, Tabria Suursoost ja Ulila luhasoost. Peale madalsoode on autor leidnud teda linaleoaugu ääres kasvavast osjastikust Meelva rabasaarelt. Iseloomulik soostunud aladele. Talvitub valmikuna.

Väga laia levikuga - Lääne-Euroopast Irkutskini. Huvitaval kombel puudub nii Soomes kui ka Rootsis.

9. Calligy pon a d u b i a (Kbm.), 1868. 3 isendit leitud Avaste soo kuivendatud aladelt. Niiskete niitude ja sooservade elanik.

Levik on ebaselge, sest varem on teda segi aetud liigiga $C$. pellucida (F.).

10. Calligypona obscurella (Boh.), 1847. Leitud samuti Avaste soo kuivendatud aladelt. Niiskete niitude elanik. Teda on samuti segi aetud liigiga $C$. pellucida (F.).

11. Callig y p on a a 1 b o c a rin at a (Stål), 1858. Usna sage madalsoodes (leitud Ogerna, Adila, Paadremaa, Nedremaa ja Epu soos), siirdesoodes (Sälliku soos) ja luhasoodes (Emajōe suudmealal). Toitub villpeal (Eriophorum vaginatum).

C. albocarinata levik on üsna ebaselge, sest varem peeti teda liigi C. distincta (Fl.) sünonüümiks. Viimane on aga temast selgesti eristatav.

12. C a 11 ig y pon a p ullul a (Boh.), 1852. (Flori (1861) Delphax pullula on Calligypona ståli (Metc.), 1943 (= Delphax bohemani Stål 1858, nec Stål 1854) (n. syn.)).

Leitud vähesel arvul mōningatest madalsoodest (Patsu-Laiküla soost, Suursoost ja väikselt Allikasoolt Otepää lähedal), luhasoodest (Järvesoo jōe luhalt, Emajōe suudmealalt) ning ka siirdesoost (Iisaku rajoonis).

Kirjanduse andmeil on iseloomulik madalsoodele.

Tuntud vaid Euroopa läänepoolsest osast.

13. Calligypona fairmairei (Perr.), 1857. (nec Delphax neglecta (Fl.) $=C$. brevipennis (Boh.) (n. syn.)) ${ }^{2}$

Esineb vähesel arvul reas madalsoodes (Avaste soos, Järise soos) ja ka Emajōe suudmeala luhasoodes. Arvukamalt esineb Avaste soo kuivendatud aladel.

Kirjanduse andmeil esineb madalsooaladel eriti mitmesugustel lugadel.

Levinud Prantsusmaalt kuni Hiinani.

${ }^{1}$ Flori kogus esineb üks ô C. pullula (Boh.) nimetuse all Delphax angusta. Kuna viimast liiki pole kusagil kirjeldatud, tuleb teda käsitada kui nomen nudum'it.

2 Oshanin (1906) ja tema järgi ka Metcalf (1943) peavad Flori Delphax neglecta't C. fairmairei sünonüümiks. Flori kogus olevad ô ồ olid aga kőik liigi C. brevipennis (Boh.) isendid. 
14. Megophth a l m us sc an icus (Fall.), 1806. Leitud üksikute eksemplaridena Saaremaal Ohtja soost ja metsasoolapikeselt ida pool Karujärve. Uksikuid isendeid leitud ka Avaste soo kuivendatud aladelt. Oldiselt kuivade alade elanik. Levinud peaaegu kogu Euroopas.

15. On o p s is tristis (Zett.), 1838. Madalsoodes laialt levinud liik. Varem loetud $O$. flavicollis'e sünonüümiks.

Levik ebaselge. Arvatavasti laia levikuga palearktiline liik.

16. Macrops is im pura (Boh.), 1845. Leitud Avaste, Paadremaa, Ogerna ja Tabria soost, Suursoost, Järvesoo jōe luhalt ja Emajôe suudmealalt, ka Avaste soo kuivendatud aladelt. Elab madalatel pajuliikidel, soodes eeskätt hundipajul (Salix rosmarinifolia). Levikualaks peaaegu kogu Euroopa.

*17. Agalli a brachyptera Boh. f. macroptera. Oks o leitud Avaste soo kuivendatud söödilt 1. VIII 1951. Autorile teadaolevail andmeil on Avaste soo pikatiiva. lise vormi kolmas leiukoht. Varem on nimetatud vormi leitud vaid Saksamaal Hannoverist (Wagner, 1936) ja Poolas Szymanovist (Smreczynski, 1954).

18. A phrodes striatus (F.) sensu. $\mathrm{Z}$ a c hw at k in, 1948, $1953=$ A. bicinctus ssp. diminutus Rib., 1952 (n, syn.).

Kõik madalsoodest leitud eksemplarid sobivad nii välistunnuste kui ka genitaalide ehituse poolest hästi Zahvatkini kirjeldustega. Kirjelduste ja eriti genitaalide jooniste vôrdlemine näitab, et 'Zahvatkini kirjeldus $A$. striatus'e kohta sobib tăiesti Ribaut' kirjeldusega ssp. diminutus'e kohta.

A. striatus'e levik ulatub Prantsusmaalt Siberini ja Kirgiisi NSV-ni.

*19. A p hrodes a lbiger (Germ.), 1821. Oksikud leiud Avaste soost ning Emajōe suudmeala luhasoodest. Eriti arvukana esines Avaste soo kuivendatud söödil (kuni 17 isendit ühes sajalöögilises kahapüügis).

Kirjanduse andmeil esineb soolakuil.

Seni leitud vaid Saksamaalt, Poolast, Tšehhoslovakkiast ja Prantsusmaalt. Esinemise kohta mujal Nõukogude Liidus puuduvad autoril andmed.

20. Strongylocephalus livens (Zett.), 1838. Leitud vaid Patsu-Laiküla soost ja soolapikeselt Riguldi juures. Sagedamini esineb Emajōe suudmeala ning Järvesoo jōe luhasoodes.

Kirjanduse järgi türfobiont. Talvitub valmikuna samblakattes.

Levikualaks peaaegu kogu Euroopa.

Nōukogude Liidust on autoril kirjanduse järgi teada veel vaid üks leid, nimelt Karjala ANSV-st.

*21. P a l u s e d wardsi (Lindb.), 1924. Seni leitud madalsoodest (Patsu-Laiküla soost, Lõõla Suursoost ja Saaremaal Karujärve soolapikeselt) ning üsna sageli Emajōe suudmeala luhasoodelt.

Kirjanduse järgi iseloomulik kôrgete tarnadega soodele.

Levik ebaselge. Selle kohta pole Nôukogude Liidu teistest osadest kirjanduses ühtki märget. Küll leidub Moskva ülikooli tsikaadidekogus üks Kesk-Uraalist pärinev isend.

*22. Jassargus (S a etus) sursumflexus (Then.), 1901. ${ }^{1}$ Eesti NSV madalsoodes laialt levinud. Harvemini esineb luhasoodes. Okoloogiliselt näib olevat seotud sinihelmikuga (Molinia coerulea), kuid otsesed vaatlused selle kohta puuduvad. Oldine levik ebaselge. Seni leitud Austriast, Inglismaalt, Hollandist, Rootsist, Soomest, Saksamaalt, Poolast, Tšehhoslovakkiast ja Sveitsist. Tema esinemisest mujal Nôukogude Liidus pole seni teateid.

*23. Sor hoanus $\mathrm{x}$ an thoneurus (Fieb.), 1869. Meie madalsoode tavalisemaid tsikaadiliike. Varem segi aetud temale lähedase $S$. assimilis'ega.

Madalsoode ja rabade elanik.

Levik ebaselge. Enamik leide pärineb Pōhja-Euroopast (Soomest, Rootsist, Norrast, Saksamaalt, TŠehhoslovakkiast, Poolast). Esineb ka Pōhja-Ameerikas (Beirne, 1954). Väljaspool Eesti NSV-d pole Nõukogude Liidus seni mujal leitud. On vôimalik, et Zahvatkini (1953, lk. 248) poolt mainitud Paludijassus kozhevnikovi A. Z. in litt. (= nom. nudum!) on identne selle liigiga; S. xanthoneurus't peab Zahvatkin (1. c.) S. assimilis'e sünonüümiks.

24. Ps a mmotettix alienus (Dhlb.), 1851. Leitud Avaste soost kuivendatud rukkipốllult 25 . VIII 1952 .

Väga laia holarktilise levikuga liik. Tuntud teravilja kahjurina. Enamik andmeid, kus kahjurina on märgitud Deltocephalus striatus, käib selle liigi kohta. ${ }^{2}$

${ }^{1}$ Flori kogus leiduvad üksikud selle liigi ('ô) eksemplarid koos $J$. flori'ga sildi all: Jassus (Deltocephalus) distinguendus lobis segm. ult. gen. inflexis.

${ }^{2}$ Nii on autor määranud seda liiki maisilt Saratovi ümbruses ning maisilt ja lutsernilt Samarkandi ümbruses Usbeki NSV-s. 
*25. P s a m m ot ettix exil is W. Wagn., 1941 (det. W. Wagner, Hamburg). Leitud vähesel arvul, kuid pidevalt Avaste soost; arvukamalt saadud Saaremaa soodest (Tohkussoost; Järise soost kuni 134 isendit ühe sajalöögilise kahapüügi kohta).

Uus liik Nōukogude Liidu faunas. Seni tuntud Saksamaalt ja Tšehhoslovakkiast.

Kirjanduse andmeil esineb vaid kserotermseis paikades (Dlabola, 1954), mille tõttu tema leidumine meie soodes on üllatav. Autori 1955. aasta nimestikus oli mainitud liik toodud ekslikult $P$. albomarginatus'e nime all.

26. P s a m motettix con $\mathrm{f}$ in is (Dhlb.), 1850. Madalsoodes esineb harva (Suursoos). Väga arvukana esineb kuivendatud aladel (kuni 225 isendit sajalöögilises kahapuugis).

Kuivade niitude liik.

Arvatavasti holarktilise levikuga.

27. Scleroracus decumanus (Kontk.), 1948. Mõned $\hat{o}$ o leitud Avaste soo kuivendatud aladelt.

Levik ebaseige. Seni leitud vaid Soomest ja Siberist (Sujetukis).

28. L imotettix atric a pill a (Boh.), 1845. Esineb vähesel arvul nii madalsoodes (Tohkussoos, Karujärve soos, Tuhu soos, Tabria Suursoos, Nōmme küla soos) kui ka luhasoodes (Järvesoo jōe luhal, Emajōe suudmealal).

Pōjapoolse levikuga liik (Rootsis, Soomes, Leningradi oblastis).

1955. a. ilmunud Kanada jassiidide faunas (Canad. Entomol., 86 (Suppl. 2):94) on see liik samastatud Põhja-Ameerika liigiga L. utahnus Laws. Nende liikide lähem vordlemine naitas, et nii välısmoríoloogias kuı ka genitaalide ehituses esineb nende vahel olulisi erinevusi, mille tõttu vōib väita, et $L$. atricapillus (Boh.) $\neq$ L. utahnus Laws.

29. Euscelis disting uendus (Kbm.), 1858. Oksikud leiud pärit 7. VIII ja 25. VIII 1952 Avaste soost kuivendatud mustalt kesalt.

Vähetuntud liik (tuntakse Saksamaalt, Poolast, Tšehhoslovakkiast, Soomest ja Karjala ANSV-st), kelle esinemist seostatakse ristikheinaga.

30. A thysan us argent a ri us Metc., 1955. Leitud üksikeksemplaridena vähestest madaisoodest (Ogerna, Patsu-Laiküla, Avaste, Paadremaa ja Mihkli soost) ning luhasoodest (Emajoe suuamealal). Esineb ka Avaste soo kuivendatud aladel. Kirjanduse järgi iseıomulik kuivemaile soostunud aladele. Levinud Kesk- ja Pōhja-Euroopas, Siberis kun Irkutskini, Turkestanis.

31. Cic adula intermedi a (Boh.), 1845. Oksik ilma tagakehata isend leitud 27. VII 1953 Järvesoo jōe luhalt. Esineb Prantsusmaal, Inglismaal, Norras, Rootsis, Soomes, Koola pooisaarel, Siberis (Irkutskis).

32. Cicadula quinquenotat a (Boh.), $1845 .^{1}$ Iseloomulik madalsoode liik. Esineb tavaliseit koos Cicadula 4-notata'ga, kuid eelistab märjemaid alasid. Puudub täiesti kuivemates madalsoodes.

Levinud Euroopa pōhjapoolses osas.

33. Macrosteles alpinus (Zett.), 1828. Uksik ô eksemplar leitud Avaste soost kuivendatud söödilt.

Linnavuori (1952) järgi monofaag ubalehel (Menyanthes trifoliata). Levikult boreoalpiinne. Seni kindlaks tehtud ühelt poolt Saksamaa, Itaalia ja Sveitsi kōrgmäestikes, teiselt poolt Soomest, Rootsist, Norrast ja Koola pooisaarelt.

34. M a crosteles crist a t us (Rib.), 1927. Esinenud Avaste soo kuivendatud aladel, ajuti (1951, a.) üsna arvukana (kuni 40 isendit sajalöögilises kahapüügis). Kaug-Idas.

Levinud peaaegu kogu Euroopas, Razvjaskina avaldamata töö (1948) andmeil ka

*35. M a crosteles $1 \mathrm{ividus} \mathrm{(Edw.),} \mathrm{1894.} \mathrm{Oksik} \mathrm{ô} \mathrm{eksemplar} \mathrm{leitud} \mathrm{Järvesoo}$ jổe luhalt 27. VII 1953.

Kirjanduse andmeil elab kōrkjatel (Scirpus) ja alssidel (Eleocharis). Seni tuntud Inglismaalt, Saksamaalt, Rootsist, Soomest ja Poolast. Peale nimetatud üksikleiu Eesti NSV-st pole autoril teada ühtki muud leidu Nōukogude Liidust.

*36. Erythroneura silvicola (Oss.), 1937. Avaste soo kuivendatud võsast leitud 28. VII 19529 ô ja 4 o isendit.

Olemasolevate andmete pōhjal näib olevat tegemist boreoalpiinse liigiga, mida seni on leitud vaid Rootsist ning Kesk-Euroopa mäestikualadelt (Śveitsist - var. alpicola Cer., Saksamaalt, Tšehhoslovakkiast, Poolast ja Austriast).

1 Flori kogus leidub rida eksemplare nimetuse all Jassus (Jassus) 4-notatus Fab. var. brevivertex m. (nomen nuduml). 
37. $\mathrm{K}$ y bos $1 \mathrm{i}$ d berg i (Linnav.), $1951=K$. betulae Zachw., $1953=$ Empoasca borealis Lindb., 1952.

Ossiannilsson (1955) on samastanud selle liigi veel liigiga Empoasca australis Wagn., 1949. Wagneri originaaldiagnoosi ja jooniste võrdlus on aga näidanud, et need on siiski kaks selgesti eristatavat liiki.

Uksikuid 우 을 kes nii suuruselt kui ka toidutaimelt sobivad antud liigi kirjeldusega, on leitud Avaste soo kuivendatud vōsast.

\title{
KIRJANDUS
}

B e i r n e, B. P., 1954. New Records and Some Synonymic for North American Leafhoppers (Homoptera: Cicadellidae). Canad. Entomol., 86 (11), lk. 495-498.

D I a bol a, J., 1954. Křísi - Homoptera. Fauna CSR 1.

Flor, C., 1861. Die Rhynchoten Livlands in systematischer Folge beschrieben. Zweiter Theil: Rhynchota gulaerostria Zett., Archiv f. Naturk. Liv-, Ehst- und Kurlands, 4.

L in d berg, H., 1947. Verzeichnis der Ostfennoskandischen Homoptera-Cicadina. Fauna Fennica 1 .

Linnavuori, R., 1952. Studies on the Ecology and Phenology of the Leafhoppers (Homoptera) of Raisio (SW Finland). Ann. Zool. Bot. Soc. Fenn. «Vanamo», $14(6)$.

Met c a 1 f, Z. P., 1943. Araeopidae. General Catalogue of the Hemiptera. Fasc. IV, Part 3.

O sh a n in, B., 1906. Verzeichnis der Palaearktischen Hemipteren. II Bd. (Homoptera).

Ossiannilsson, F., 1951. Homoptera aus einigen nordestländischen Inseln. Opuscula entomologica, 16 (1), lk. 10-14.

O ss i a nnilss on, F., 1955. Några för Sverige nya stritar (Hom. Auchennorrh.) med en synonymisk anmärkning. Entomol. Tidskr., 76 (2-4), lk. 131-133.

Ра з в яз к н н а Г. M., 1948. Систематика и экология цикадок рода Macrosteles и их значение в распространении вирусных заболеваний растений. Диссертация (рукопись). Москва.

R ib a ut, H., 1952. Homopteres Auchenorhynques II, (Jassidae). Faune de France 57.

S mreczynski, S., 1954. Materialy do faunu pluskwiaków (Homoptera) Polski. Fragmenta Faunistica, 7 (1), 1k. 1-146.

Vil b a s te, J., 1955. Eesti NSV soode rohurinde nokaliste faunast. Loodusuurijate Seltsi Aastaraamat, 48, lk. 104-121.

W a g n e r, W., 1936. Neue Homoptera-Cicadina aus Norddeutschland. Verh. Ver. naturw. Heimat f., Hamburg 25, lk. 69-73.

W a g n er, W., 1949. Drei neue Typhlocybiden aus Steiermark. Zb. Gesamtgeb. Entomol., $3,1 \mathrm{k} .43-45$.

3 а х в а т к и н А. А., 1948., Новые и малоизвестные цикадины из Окского заповедника. Научно-метод. зап. 11, lk. 186-197.

3 а х в а тк и н А. А., 1953. Сборник научных трудов. Изд. МГУ.
Eesti NSV Teaduste Akadeemia
Zooloogia ja Botaanika Instituut
Saabus toimetusse
8. V 1957

\section{ЗАМЕТКИ К ФАУНЕ ЦИКАД НИЗИННЫХ БОЛОТ ЭСТОНСКОЙ ССР}

\author{
ю. Г. Вильбасте
}

\section{Резюме}

В статье приводятся данные о нанболее интересных видах цикад, обнаруженных при исследовании энтомофауны низинных болот Эстонской ССР. Все приводимые виды (за нсключением Aphrodes striatus (F.)) являются новыми для Әстонии. Девять видов, отмеченные в тексте основной статьи звездочками, являются новыми для всего Советского Союза. Выяснены следующие новые синонимы:

Delphax pullula Flor, $1861=$ Calligypona ståli (Metc.), 1943;

Delphax neglecta Flor, $1861=$ Calligypona brevipennis (Boh.), 1847; nec C. fairmairei

(Perr.), 1857; 
Aphrodes bicinctus ssp. diminutus Rib., $1952=$ Aphrodes striatus (F.) s. Zachwatkin, 1948, 1953;

Kybos betulae Zachw., $1953=$ Kybos lindbergi (Linnav.), $1951=$ Empoasca borealis Lindb., 1952; nec E. australis Wagn., 1948.

Институт зоологии и ботаники

Академии наук Эстонской ССР
Поступила в редакцию 8 V 1957

\section{BEMERKUNGEN ZUR ZIKADENFAUNA DER NIEDERUNGSMOORE IN DER ESTNISCHEN SSR}

\section{J. Wilbaste}

\section{Zusammenfassung}

Es werden im Artikel die interessantesten Zikadenarten behandelt, die bei einer entomologischen Untersuchung estnischer Niederungsmoore gefunden worden sind. Sämtliche Arten (mit Ausnahme von Aphrodes striatus (F.)) sind für die Estnische SSR neu. Neun mit einem Sternchen bezeichnete Arten sind neu für die ganze Sowjetunion. Als neue Synonyme werden angegeben:

Delphax pullula Flor, 1861 = Calligypona ståli (Metc.), 1943;

Delphax neglecta Flor, 1861 = Calligypona brevipennis (Boh.), 1847; nec C. fairmairei (Perr.), 1857;

Aphrodes bicinctus ssp. diminutus Rib., 1952=Aphrodes striatus (F.) s. Zachwatkin, 1948, 1953;

Kybos betulae Zachw., $1953=$ Kybos lindbergi (Linnav.), $1951=$ Empoasca borealis Lindb., 1952; nec E. australis Wagn., 1948.

Institut für Zoologie und Botanik der Akademie der Wissenschaften der Estnischen SSR
Eingegangen am 8. Mai 1957 